\title{
Creating Weyl nodes and controlling their energy by magnetization rotation
}

\author{
Madhav Prasad Ghimire,,${ }^{1,2,3}$ Jorge I. Facio $\odot,{ }^{1}$ Jhih-Shih You, ${ }^{1}$ Linda Ye, ${ }^{4}$ Joseph G. Checkelsky, ${ }^{4}$ Shiang Fang, ${ }^{5}$ \\ Efthimios Kaxiras, ${ }^{5,6}$ Manuel Richter, ${ }^{1,7}$ and Jeroen van den Brink ${ }^{1}$ \\ ${ }^{1}$ Institute for Theoretical Solid State Physics, IFW Dresden, Helmholtzstrasse 20, 01069 Dresden, Germany \\ ${ }^{2}$ Central Department of Physics, Tribhuvan University, Kirtipur, 44613, Kathmandu, Nepal \\ ${ }^{3}$ Condensed Matter Physics Research Center, Butwal-11, Rupandehi, Nepal \\ ${ }^{4}$ Department of Physics, Massachusetts Institute of Technology, Cambridge, Massachusetts 02139, USA \\ ${ }^{5}$ Department of Physics, Harvard University, Cambridge, Massachusetts 02138, USA \\ ${ }^{6}$ John A. Paulson School of Engineering and Applied Sciences, Harvard University, Cambridge, Massachusetts 02138, USA \\ ${ }^{7}$ Dresden Center for Computational Materials Science (DCMS), TU Dresden, 01069 Dresden, Germany
}

(Received 11 March 2019; revised manuscript received 18 October 2019; published 26 December 2019)

\begin{abstract}
As they do not rely on the presence of any crystal symmetry, Weyl nodes are robust topological features of an electronic structure that can occur at any momentum and energy. Acting as sinks and sources of Berry curvature, Weyl nodes have been predicted to strongly affect the transverse electronic response, like in the anomalous Hall or Nernst effects. However, to observe large anomalous effects the Weyl nodes need to be close to or at the Fermi level, which implies the band structure must be tuned by an external parameter, e.g., chemical doping. Here we show that in a ferromagnetic metal tuning of the Weyl node energy and momentum can be achieved by rotation of the magnetization. First, taking as example the elementary magnet hcp-Co, we use electronic structure calculations based on density-functional theory to show that by canting the magnetization away from the easy axis, Weyl nodes can be driven exactly to the Fermi surface. Second, we show that the same phenomenology applies to the kagome ferromagnet $\mathrm{Co}_{3} \mathrm{Sn}_{2} \mathrm{~S}_{2}$, in which we additionally show how the dynamics in energy and momentum of the Weyl nodes affects the calculated anomalous Hall and Nernst conductivities. Our results highlight how the intrinsic magnetic anisotropy can be used to engineer Weyl physics.
\end{abstract}

DOI: 10.1103/PhysRevResearch.1.032044

Materials hosting unconventional quasiparticles, such as Weyl semimetals, constitute a framework with potential for novel electronic devices. One ground for such expectation is the possibility of enhancing the response to external fields by engineering topological properties of the electronic states. For a material to specifically host Weyl fermions, the spin degeneracy of the electronic bands has to be removed by breaking either time-reversal symmetry $(\Theta)$ or inversion. Karplus and Luttinger [1] first noticed that in a $\Theta$-broken system the spin-orbit coupling can introduce in the manifold of Bloch states a left-right asymmetry which in turn, in the presence of an electric field, causes a Hall current at zero magnetic field. This scattering-independent mechanism originates in the so-called anomalous velocity of the wave packets, which can be written in terms of the Berry curvature of the Bloch states in momentum space. Weyl nodes are monopoles of Berry curvature, which implies first that they can only be created and annihilated in pairs of opposite monopole charge and second that wave packets made out of Weyl fermions can have a large anomalous velocity. As this velocity is perpendicular to the

Published by the American Physical Society under the terms of the Creative Commons Attribution 4.0 International license. Further distribution of this work must maintain attribution to the author(s) and the published article's title, journal citation, and DOI. electric field, Weyl systems can exhibit enhanced transverse electronic responses, as in the Hall or Nernst effects.

This effect has been argued to be at work in different materials in which the anomalous velocity contribution intrinsic to the band structure is at the heart of enhanced electric and thermoelectric performance in the regimes of both linear [2-5] and nonlinear [6,7] response. Still, a central problem for optimizing Berry-curvature-based effects is the energy of the Weyl nodes, which currently is not a controlled variable from a material design point of view. Indeed, as the only symmetry restriction is to break inversion or $\Theta$, Weyl nodes can occur at any momentum and energy [8-10]. The interplay with the symmetry enables interesting effects of external magnetic fields as, e.g., using the field to split symmetry-protected fourfold crossing to create Weyl nodes [11-16]. In this work, we analyze a different interplay between magnetism and topology, one that does not rely on the existence of such kind of crossings: We explore the effects of changing the orientation of the magnetization $(\mathbf{m})$ on the electronic structure topology of ferromagnets. Rotation of $\mathbf{m}$ can be achieved by changing thermodynamic variables such as external magnetic fields [17], temperature [18,19], hydrostatic pressure [20], and uniaxial strain [21].

Here, we show that the magnetization reorientation can be used as a strategy to tune Weyl nodes to the Fermi surface. We consider two examples, the elementary magnet hcp-Co and the half-metal $\mathrm{Co}_{3} \mathrm{Sn}_{2} \mathrm{~S}_{2}$, a ferromagnet recently found to be a Weyl semimetal and to display a large anomalous Hall effect 

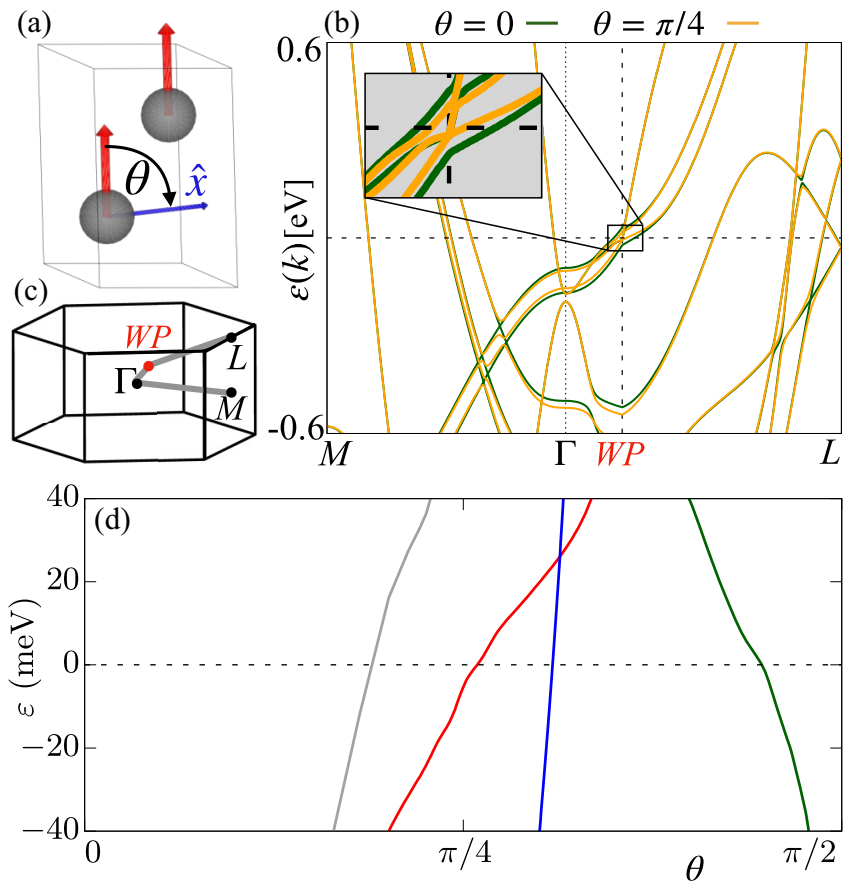

FIG. 1. (a) Crystal structure of hcp-Co and definition of $\theta$. (b) GGA band structure with the magnetization along the easy axis $(\theta=0)$ or along $\theta=\pi / 4$. The Fermi level at zero energy is indicated by a dotted line. (c) Brillouin zone. The red point is a Weyl node close to the Fermi level at $\theta=\pi / 4$ and has coordinates $\sim 2 \pi / a[0.088,0,0.104]$. (d) Energy of four specific Weyl nodes as a function of $\theta$. Different colors correspond to sets of Weyl nodes degenerated by symmetry.

(AHE) [22-25]. Based on density functional theory (DFT), we show that canting $\mathbf{m}$ away from the easy axis leads to the creation of new Weyl nodes and to large displacements of the Weyl points in energy and in momentum space such that, at specific orientations, they can be placed exactly at the Fermi surface. Furthermore, we analyze how this tuning affects the anomalous Hall conductivity (AHC) and its thermal counterpart, the anomalous Nernst conductivity (ANC). Calculations as a function of the canting angle reveal that the ANC in particular is highly susceptible to the Weyl dynamics: It displays sharp peaks when asymmetric hole and electron pockets exist nearby the band crossing that reaches the Fermi energy.

Controlling Weyl nodes in an elementary magnet. We start our discussion with hcp-Co (space group 164), a ferromagnet with a magnetic anisotropy energy (MAE) of $65 \mu \mathrm{eV}$ per Co in favor of the easy-axis configuration [26,27]. The magnetic field required to put the magnetic moments in plane is less than 2 Tesla [28].

We performed DFT calculations of the ferromagnetic phase with $\mathbf{m}$ in the $x z$ plane at an angle $\theta$ from $\hat{z}$ [29]. Figure 1(b) presents the band structure for the cases $\theta=$ $0, \pi / 4$. The rotation of $\mathbf{m}$ induces appreciable changes in the splitting between some of the bands, and even makes some band crossings be avoided and vice versa. We searched for Weyl nodes in a low-energy window $[-40,40] \mathrm{meV}$ following their trajectories as a function of $\theta$ [30]. Remarkably, at specific angles, Weyl nodes are exactly at the Fermi surface

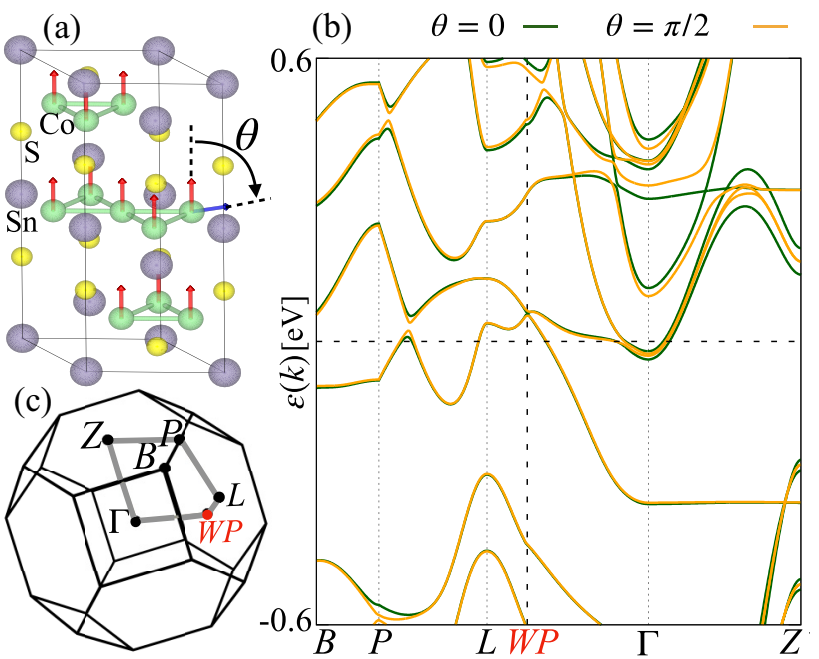

FIG. 2. (a) Crystal structure of $\mathrm{Co}_{3} \mathrm{Sn}_{2} \mathrm{~S}_{2}$. (b) Band structure with the magnetization along the easy axis $(\theta=0)$ or in the plane $(\theta=\pi / 2)$. The Fermi level at zero energy is indicated by a dotted line. (c) Brillouin zone. The red point corresponds to one of the Weyl nodes at $\theta=0$ and has coordinates $\sim 2 \pi / a[0.48,0,0.08]$.

[Fig. 1(d)]. Of particular interest is the case $\theta \sim \pi / 4$, in which the nodes crossing the Fermi energy form the minimum set of only two points lying in the LГM plane (red line).

It is interesting to note that while different exchange and correlation functionals yield significantly different MAE [the generalized gradient approximation (GGA) results in $8 \mu \mathrm{eV}$ while GGA plus the orbital polarization correction gives $150 \mu \mathrm{eV}]$, calculations based on both functionals result in similar Weyl-node energy trajectories [31]. This shows that the energy change of the Weyl nodes need not scale proportionally to the MAE and, therefore, that analogous tuning of the Weyl nodes to the Fermi surface can occur in magnets with significantly different MAE. It is naturally of interest to analyze if such tuning is also possible in materials with a low-energy band structure simpler than that of hcp-Co. Therefore, we focus now on the Weyl semimetal $\mathrm{Co}_{3} \mathrm{Sn}_{2} \mathrm{~S}_{2}$.

The half-metal $\mathrm{Co}_{3} \mathrm{Sn}_{2} \mathrm{~S}_{2}$. This system presents a ferromagnetic phase of Curie temperature $T_{C}=172 \mathrm{~K}$ [32]. The crystal structure has space group $R-3 m$ (no. 166) with lattice parameters $a=5.37 \AA$ and $c=13.18 \AA$ and consists of quasitwo-dimensional (quasi-2D) $\mathrm{Co}_{3} \mathrm{Sn}$ layers separated by $\mathrm{S}_{2} \mathrm{Sn}$ layers with the particularity that the stack of cobalt atoms forms a kagome lattice; see Fig. 2(a). Recently, different groups $[22,23]$ have reported in the magnetic ground state six Weyl nodes lying $60 \mathrm{meV}$ above the Fermi energy. Similar to hcp-Co, the rotation of $\mathbf{m}$ induces appreciable changes in electronic structure [see Fig. 2(b)]. The ground state corresponds to $\theta=0$, with a magnetic moment of $0.33 \mu_{B}$, which agrees well with magnetization measurements, $0.29 \mu_{B}$ [33] or $0.31 \mu_{B}$ [32]. The calculated MAE is $\approx 0.19 \mathrm{meV}$ per Co atom. The field required to rotate the moment into the plane at zero temperature is estimated to be $\approx 26$ Tesla $[28,31]$.

Interestingly, the number of Weyl points in the energy range $[-100,150] \mathrm{meV}$ increases from 6 to 26 as $\theta$ changes from 0 to $\pi / 2$. We find two regimes in the evolution of the 

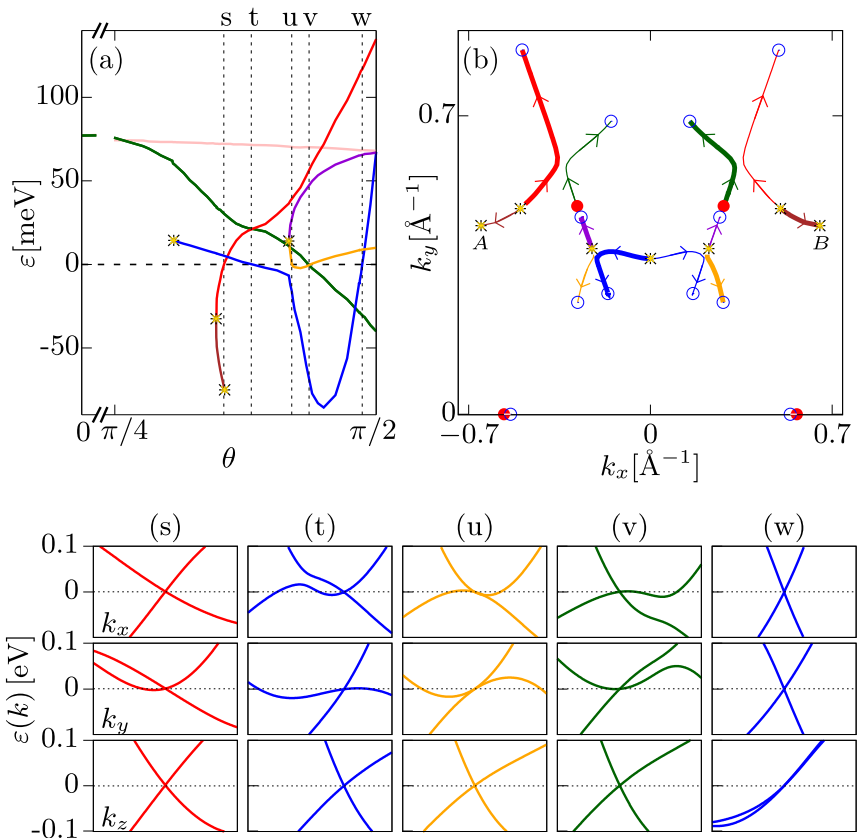

FIG. 3. Weyl nodes dynamics. (a) Energy of the Weyl nodes as a function of $\theta$. Different colors correspond to sets of Weyl points of different energy. The yellow marks signal the creation or annihilation of Weyl nodes. Vertical lines indicate angles (labeled s, t,..., w) at which Weyl fermions place at the Fermi surface. (b) Projection of Weyl node trajectories on the semiplane $k_{z}=0$ with $k_{y} \geqslant 0$. Filled red and empty blue circles correspond to Weyl points at $\theta=0$ and $\theta=\pi / 2$, respectively. The thin (thick) lines indicate the trajectories of Weyl points of positive (negative) chirality and follow the color code of panel (a) while the arrows indicate the sense of motion as $\theta$ increases. Weyl points $A$ and $B$ annihilate each other by approaching the Brillouin zone border when their separation reaches a reciprocal lattice vector. (s)-(w) For the angles in which a Weyl node crosses the chemical potential, energy dispersion along paths centered at the corresponding Weyl point. The paths have length $2 \pi /(5 a)$ and the rows from top to bottom correspond to $k_{x}, k_{y}$, and $k_{z}$, respectively.

band structure as $\mathbf{m}$ goes from out of plane to in plane. The first occurs at small angles and consists in the splitting in energy of the original six Weyl points due to the breaking of the $\mathrm{C}_{3}$ symmetry. Namely, the nonmagnetic point symmetry group is $D_{3 d}(b)$ [31] and a finite $\mathbf{m}$ breaks $\Theta$ and affects some of the unitary symmetry operations. In total, for $\mathbf{m} \| \hat{z}$, there are 12 point symmetry operations and the application of these to a Weyl node that lies in the $k_{y}=0$ plane leads to six degenerated Weyl nodes. Upon canting $\mathbf{m}$ at an angle $\theta$ towards $\hat{x}$, the only remaining symmetries - in addition to the identity and to inversion-are $\Theta \cdot C_{2}(y)$ and $\Theta \cdot M(y)$. This forces the six Weyl nodes to split in energy as a doublet (formed by those with $k_{y}=0$ ) and a quartet. Figure 3(a) shows the calculated energy of the Weyl nodes as a function of $\theta$. For $\theta<\pi / 4$, this splitting is smaller than $4 \mathrm{meV}$.

Further increasing $\theta$, the second regime is signaled by large changes in the Weyl-node structure. In particular, the Weyl-node quartet goes from above to below the Fermi level and new Weyl nodes are created. Notice that in this angle range, the symmetry does not change. Therefore, the Weylnode creation is not due to the splitting of a Dirac point as in
Ref. [11-16]. The vertical lines in Fig. 3(a) indicate the angles (labeled s, t, . ., w) at which a Weyl node crosses the Fermi surface. The remarkable changes in energy go hand in hand with large displacements in momentum space. Figure 3(b) shows the projection of the trajectories on the semiplane $k_{z}=$ 0 with $k_{y} \geqslant 0$, illustrating the rich dynamics controlled by $\theta$. As shown in Figs. 3(s)-3(w), the energy dispersion close to the nodes reaching the Fermi energy can differ significantly among the different cases [34].

Experimental consequences. We analyze next observables that can make evident the rich Weyl dynamics induced by rotating $\mathbf{m}$. We focus on response functions that originate in the Berry curvature of the Bloch states through the anomalous velocity [35]. We compute the Hall response to an electric field $\mathcal{E}$ or to a temperature gradient $\nabla T$ at zero external magnetic field. In the linear response, the Hall current is $j_{a}=\sigma_{a b} \mathcal{E}_{b}-\alpha_{a b} \nabla_{b} T$, where $\sigma_{a b}$ and $\alpha_{a b}$ read

$$
\left\{\sigma_{a b}, \alpha_{a b}\right\}=\sum_{n, c} \epsilon_{a b c} \int \frac{d^{3} \mathbf{k}}{(2 \pi)^{3}} \Omega_{n, c}(\mathbf{k})\left\{\frac{-e^{2}}{\hbar} f_{0}\left(E_{n \mathbf{k}}\right), s\left(E_{n \mathbf{k}}\right)\right\} .
$$

Here, $\epsilon_{a b c}$ is the Levi-Civita tensor, $E_{n \mathbf{k}}$ is the energy dispersion of the $n$th band, $\Omega_{n, c}$ is the $c$ component of the Berry curvature, and $\boldsymbol{\Omega}_{n}(\mathbf{k})=\nabla_{\mathbf{k}} \times \mathbf{A}_{n}(\mathbf{k})$, where $\mathbf{A}_{n}(\mathbf{k})=$ $-i\left\langle u_{n \mathbf{k}} \mid \partial_{\mathbf{k}} u_{n \mathbf{k}}\right\rangle$ [36], $f_{0}$ is the equilibrium Fermi distribution, $s\left(E_{n \mathbf{k}}\right)=f_{0}\left(E_{n \mathbf{k}}\right)\left(E_{n \mathbf{k}}-\mu\right) / T-k_{B} \log \left(1-f_{0}\left(E_{n \mathbf{k}}\right)\right)$ is the entropy density, and $\mu$ the chemical potential [37-46]. As $T \rightarrow 0 \mathrm{~K}$, the entropy vanishes and so does $\alpha_{a b}$, while at finite $T, s\left(E_{n \mathbf{k}}\right)$ is maximum at $\mu$ and decreases exponentially away from the Fermi surface. Joint measurements of electric and thermoelectric coefficients can determine $\sigma_{a b}$ and $\alpha_{a b}$ [47].

Figures 4(a) and 4(b) show the calculated AHCs as a function of $\theta$ [48]. For $\theta=0$, only $\sigma_{x y}$ is nonzero and its very large value $1200(\Omega \mathrm{cm})^{-1}$ is in good agreement with values reported of $1310(\Omega \mathrm{cm})^{-1}$ [23] and $1100(\Omega \mathrm{cm})^{-1}[22]$. When $\mathbf{m}$ acquires a finite projection on $\hat{x}$, the only symmetry involving a mirror plane is $\Theta M(y)$ and both $\sigma_{x y}$ and $\sigma_{y z}$ are symmetry allowed. Namely, under mirror symmetry, $\boldsymbol{\Omega}$ behaves as a pseudovector so that $M(y) \Omega_{y}(\mathbf{k})=\Omega_{y}\left(k_{x},-k_{y}, k_{z}\right)$ while $M(y) \Omega_{\|}(\mathbf{k})=-\Omega_{\|}\left(k_{x},-k_{y}, k_{z}\right)$, where $\Omega_{\|}$refers to the components of $\boldsymbol{\Omega}$ along $\hat{x}$ and $\hat{z}$ (parallel to the mirror plane). Therefore, if $\Theta M(y)$ is a symmetry, the Berry curvature satisfies $\Omega_{y}(\mathbf{k})=-\Omega_{y}\left(-k_{x}, k_{y},-k_{z}\right)$ and $\Omega_{\|}(\mathbf{k})=$ $\Omega_{\|}\left(-k_{x}, k_{y},-k_{z}\right)$ and these constraints only make $\sigma_{x z}$ vanish.

As $\theta$ increases, $\sigma_{x y}$ and $\sigma_{y z}$ follow opposite trends and at $\theta=\pi / 2, \sigma_{y z} \sim 200(\Omega \mathrm{cm})^{-1}$ is much larger than $\sigma_{x y}$. At this angle, the $x y$ component does not vanish as it is still symmetry allowed. The smallness of its value is still related to the effects of the symmetries on the Berry curvature flux. In this compound, the large AHC arises mainly from the nodal lines that become gapped when spin-orbit coupling is included. When $\mathbf{m}$ points along $\hat{z}$, the combination of $C_{3}$ and the mirror planes $\Theta M(x), \Theta M(y)$ forces the different patches of the nodal lines to contribute additively to the flux of $\Omega_{z}$ [31]. When $\mathbf{m}$ acquires a component along $\hat{x}$, the reduction in the symmetries removes this constraint and large cancelations occur due to the opposite flux of $\Omega_{z}$ at different points $\left(k_{x}, k_{y}\right)$. This geometrical effect controls the overall evolution of $\sigma_{x y}$ 

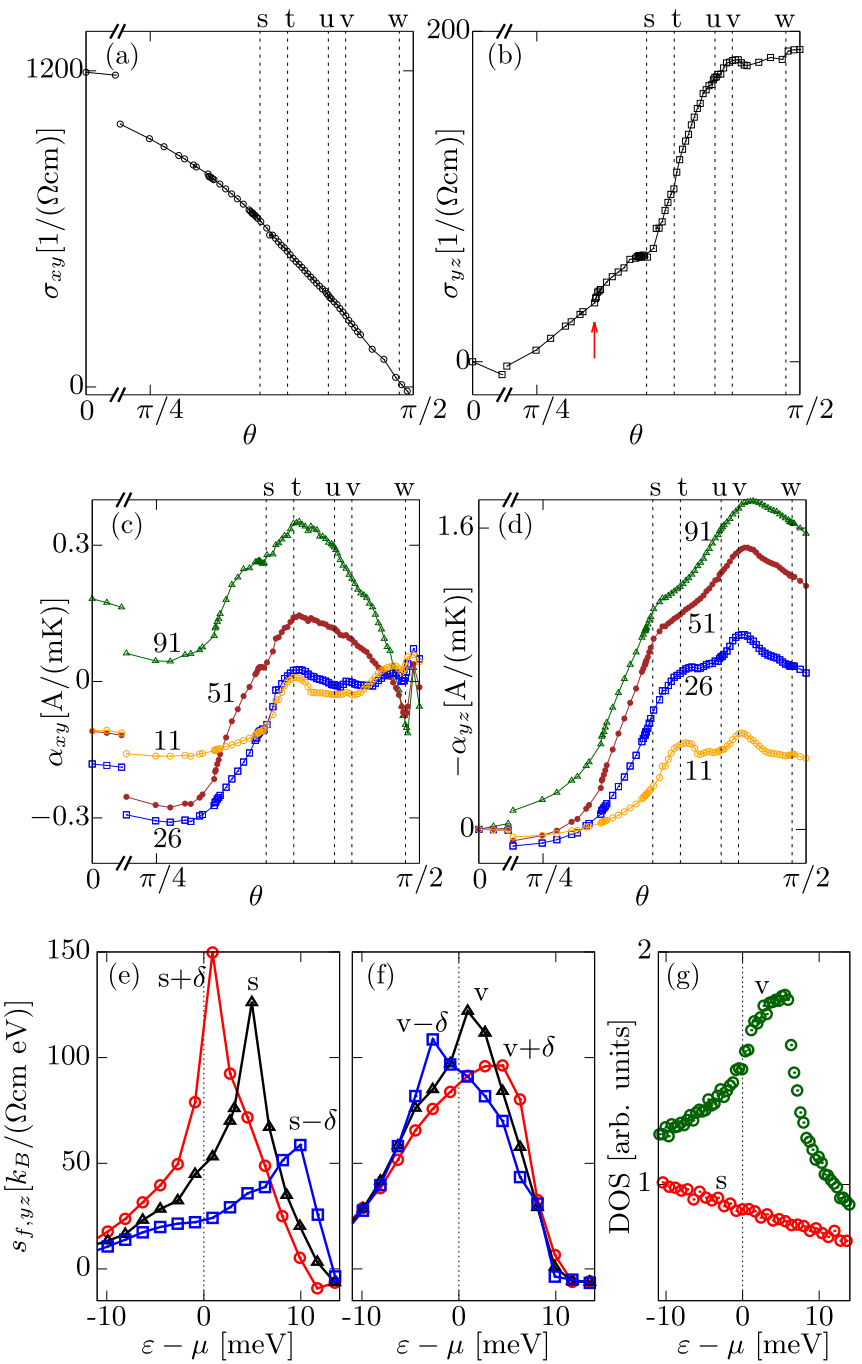

FIG. 4. [(a), (b)] Anomalous Hall conductivity ( $x y$ and $y z$ components) at zero temperature as a function of $\theta$. The red arrow indicates an angle at which pairs of Weyl nodes are created. [(c), (d)] Nernst conductivity as a function of $\theta$ for different temperatures (indicated in units of $\mathrm{K}$ for each curve). Note that $\alpha_{y z}$ is multiplied with -1 . (e) Entropy-flow density as a function of energy for $\theta=\mathrm{s}, \mathrm{s} \pm \delta$ with $\delta \sim 4^{\circ}$. (f) Entropy-flow density for $\theta=\mathrm{v}$, $\mathrm{v} \pm \delta$. The data correspond to the $y z$ component and at $T=51 \mathrm{~K}$. (g) DOS as a function of energy associated with the states close to the corresponding Weyl nodes at the Fermi surface for $\theta=\mathrm{s}$ and $\mathrm{v}$.

which is, therefore, quite smooth and monotonous. $\sigma_{y z}$ lacks this geometrical effect and, as a result, is more sensitive to detailed changes in the band structure and Weyl dynamics, as shown in Fig. 4(b). For instance, the red arrow in the plot indicates an angle at which the creation of Weyl nodes leads to a steplike increase of $\sigma_{y z}$. As $\theta$ increases further, a set of Weyl nodes reaches the Fermi energy at $\theta=\mathrm{s}$ and $\sigma_{y z}$ exhibits a small plateau. The energy dispersion of these Weyl fermions is shown in Fig. 3(s) and makes clear that this node is type I, and therefore the observed plateau is consistent with the general prediction for such Weyl nodes when their energy approaches the Fermi level [49].

Admittedly, the resulting changes in the AHC related to the Weyl nodes are not large. It turns out that the ANC shows much larger changes when Weyl nodes approach the Fermi energy. Figures 4(c) and 4(d) show the ANCs as a function of $\theta$ for different values of $T$. Since thermal fluctuations of the Co magnetic moments are not considered in Eq. (1), we restrict our analysis to a range of $T$ where the magnetization is essentially saturated $(T<100 \mathrm{~K})$ [32]. Remarkably, the dependence on $\theta$ is nonmonotonous and includes sharp peaks at specific angles. The peaks are centered at some of the angles in which a Weyl node crosses the Fermi energy (specifically, $\theta=\mathrm{t}$ and $\mathrm{v}$ ) and become broader as $T$ increases. The nonmonotonicity remains, however, clearly visible for all temperatures. The magnitude of the enhancements is worth noting: At $91 \mathrm{~K}$, starting from $\approx 0.1 \mathrm{~A}(\mathrm{mK})^{-1}$ at $\pi / 4,\left|\alpha_{y z}\right|$ reaches a maximum $1.8 \mathrm{~A}(\mathrm{mK})^{-1}$. This is five times the maximum obtained in $\mathrm{Mn}_{3} \mathrm{Sn}[47,50]$ and half of the giant $\mathrm{ANC}$ in $\mathrm{Co}_{2} \mathrm{MnGa}$ [51].

Entropy flow and Weyl fermions. To understand why and when a peak in the ANC occurs, it proves useful to analyze how different carriers contribute to the ANC. Since in a fermionic system the available entropy is restricted to states of energy $\approx k_{B} T$ around $\mu$, we are interested in resolving the contribution of different electronic states within this thermal energy window. We recast the ANC as

$$
\alpha_{a b}=-\frac{1}{e} \int_{-\infty}^{\infty} d \varepsilon \frac{\partial \sigma_{a b}}{\partial \varepsilon} s(\varepsilon)
$$

(see Ref. [31]). This equation has the overall shape of the Mott relation-involving the derivative with respect to energy of the AHC and the entropy-but holds for larger temperature and motivates us to define the entropy-flow density per energy, $s_{f, a b}(\varepsilon)=\frac{\partial \sigma_{a b}}{\partial \varepsilon} s(\varepsilon)$. The entropy flow $s_{f, a b}(\varepsilon)$ measures the contribution to $\alpha_{a b}$ of carriers of different energy and, therefore, its calculation allows us to compare the contribution of different sets of Weyl points.

We focus on $\alpha_{y z}$ and first analyze $\theta=$ s. Figure 4(e) presents $s_{f, y z}(\varepsilon)$ for $\theta=\mathrm{s}$ and for two other angles close to it, $\mathrm{s} \pm \delta$ with $\delta \sim 4^{\circ}$. For each angle, the entropy flow presents a peak as a function of energy which highlights the energy of the carriers that contribute the most to the ANC. Notice that this peak does not need to be centered at the energy of maximum entropy $(\varepsilon=\mu)$. For instance, at $\theta=\mathrm{s}-\delta$ the largest contribution is found $\approx 10 \mathrm{meV}$ above $\mu$. As $\theta$ increases, this peak achieves a larger value and its position shifts to smaller energy in a rather monotonous way. In particular, at $\theta=\mathrm{s}$ the contribution of carriers at the Fermi surface is markedly smaller than that of the states conforming the entropy-flow peak. This explicitly shows that the set of Weyl nodes crossing the Fermi energy at this angle does not contribute significantly to the ANC.

The situation is different at angles $\theta=\mathrm{t}$ and $\mathrm{v}$. Figure 4(f) shows $s_{f, y z}(\varepsilon)$ for $\theta=\mathrm{v}$ and $\theta=\mathrm{v} \pm \delta$ and makes clear that the entropy-flow peak is at the chemical potential when the Weyl nodes reach the Fermi surface $(\theta=\mathrm{v})$. This indicates that in this case the Weyl points indeed dominate the ANC.

The different contribution of different Weyl nodes to the ANC can be traced to the band structure close to the Weyl nodes [see Figs. 3(s), 3(t) and 3(v)]. While at $\theta=\mathrm{s}$ the Weyl point at the Fermi surface is type I with only a small tilt, at angles $\theta=\mathrm{t}$ and $\mathrm{v}$ the dispersion of one of the bands involved 
in the crossing at the Fermi level is such that it produces asymmetric electron and hole pockets close to the node. To illustrate this, Fig. 4(g) shows that the density of states (DOS) in a sphere of radius $\pi /(5 a)$ centered at the corresponding Weyl node at the Fermi surface is larger and more electronhole asymmetric for $\theta=\mathrm{v}$ than for $\mathrm{s}$. In an analagous way, for $\theta=\mathrm{v}$, the more asymmetric distribution of states close to the nodes at the Fermi surface contributes to a larger $\partial \sigma_{y z} / \partial \varepsilon$ and hence a larger entropy flow. We thus associate the enhanced ANC at some of the angles at which a Weyl node reaches the Fermi surface with having asymmetric hole and electron pockets close to the Weyl point at the Fermi energy.

In summary, we have shown that in ferromagnets a rich dynamics of Weyl points results from a change in the direction of the magnetization: Weyl nodes can be created, annihilated, and shifted in energy-momentum space over large distances. This dynamics can be used to place Weyl nodes exactly at the Fermi surface, which can lead to sharp enhancements in the anomalous Nernst conductivity that survive up to relatively high temperatures. Whereas we established the effect of rotation of the magnetization on Weyl nodes in the elementary magnet hcp-Co and in the semimetal $\mathrm{Co}_{3} \mathrm{Sn}_{2} \mathrm{~S}_{2}$, the finding that the energy change of the Weyl nodes does not need to scale proportionally to the magnetic anisotropy energy suggests that similar tuning of Weyl nodes to the Fermi surface can be expected in a wide set of ferromagnets. This provides a pathway to experimentally probe, manipulate, and control Weyl-fermion transport properties in magnetic Weyl semimetals.

Acknowledgments. We thank Ulrike Nitzsche for technical assistance. M.R. and J.v.d.B. acknowledge support from the German Research Foundation (DFG) via SFB 1143, Project A5. M.P.G. and J.I.F. thank the Alexander von Humboldt Foundation for financial support through the Georg Forster Research Fellowship Program. J.I.F. and J.-S.Y. thank the IFW excellence programme. J.G.C. acknowledges support by the Betty Moore Foundation EPiQS Initiative, Grant No. GBMF3848 and the United States-Israel Binational Science Foundation (BSF, Grant No. 2016389). L.Y. acknowledges support by the Tsinghua Education Foundation. E.K. and S.F. were supported by the STC Center for Integrated Quantum Materials, NSF Grant No. DMR-1231319, and by ARO MURI Award No. W911NF-14-0247.

M.P.G. and J.I.F. contributed equally to this work.
[1] R. Karplus and J. M. Luttinger, Hall effect in ferromagnetics, Phys. Rev. 95, 1154 (1954).

[2] S. Nakatsuji, N. Kiyohara, and T. Higo, Large anomalous Hall effect in a non-collinear antiferromagnet at room temperature, Nature (London) 527, 212 (2015).

[3] T. Suzuki, R. Chisnell, A. Devarakonda, Y.-T. Liu, W. Feng, D. Xiao, J. W. Lynn, and J. G. Checkelsky, Large anomalous Hall effect in a half-Heusler antiferromagnet, Nat. Phys. 12, 1119 (2016).

[4] A. K. Nayak, J. E. Fischer, Y. Sun, B. Yan, J. Karel, A. C. Komarek, C. Shekhar, N. Kumar, W. Schnelle, J. Kübler, C. Felser, and S. S. P. Parkin, Large anomalous Hall effect driven by a nonvanishing Berry curvature in the noncolinear antiferromagnet $\mathrm{Mn}_{3} \mathrm{Ge}$, Sci. Adv. 2, e1501870 (2016).

[5] N. J. Ghimire, A. S. Botana, J. S. Jiang, J. Zhang, Y.-S. Chen, and J. F. Mitchell, Large anomalous Hall effect in the chiral-lattice antiferromagnet $\mathrm{CoNb}_{3} \mathrm{~S}_{6}$, Nat. Commun. 9, 3280 (2018).

[6] L. Wu, S. Patankar, T. Morimoto, N. L. Nair, E. Thewalt, A. Little, J. G. Analytis, J. E. Moore, and J. Orenstein, Giant anisotropic nonlinear optical response in transition metal monopnictide Weyl semimetals, Nat. Phys. 13, 350 (2017).

[7] J. I. Facio, D. Efremov, K. Koepernik, J.-S. You, I. Sodemann, and J. Van den Brink, Strongly Enhanced Berry Dipole at Topological Phase Transitions in BiTeI, Phys. Rev. Lett. 121, 246403 (2018).

[8] N. P. Armitage, E. J. Mele, and A. Vishwanath, Weyl and Dirac semimetals in three-dimensional solids, Rev. Mod. Phys. 90, 015001 (2018).

[9] M. Z. Hasan, S.-Y. Xu, I. Belopolski, and S.-M. Huang, Discovery of Weyl fermion semimetals and topological
Fermi arc states, Annu. Rev. Condens. Matter Phys. 8, 289 (2017).

[10] B. Yan and C. Felser, Topological materials: Weyl semimetals, Annu. Rev. Condens. Matter Phys. 8, 337 (2017).

[11] A. A. Burkov, M. D. Hook, and L. Balents, Topological nodal semimetals, Phys. Rev. B 84, 235126 (2011).

[12] T. Liang, Q. Gibson, M. N. Ali, M. Liu, R. J. Cava, and N. P. Ong, Ultrahigh mobility and giant magnetoresistance in the dirac semimetal $\mathrm{Cd}_{3} \mathrm{As}_{2}$, Nat. Mater. 14, 280 (2015).

[13] C.-Z. Li, L.-X. Wang, H. Liu, J. Wang, Z.-M. Liao, and D.-P. $\mathrm{Yu}$, Giant negative magnetoresistance induced by the chiral anomaly in individual $\mathrm{Cd}_{3} \mathrm{As}_{2}$ nanowires, Nat. Commun. 6, 10137 (2015).

[14] M. Hirschberger, S. Kushwaha, Z. Wang, Q. Gibson, S. Liang, C. A. Belvin, B. A. Bernevig, R. J. Cava, and N. P. Ong, The chiral anomaly and the thermopower of Weyl fermions in the half-Heusler GdPtBi, Nat. Mater. 15, 1161 (2016).

[15] J. Cano, B. Bradlyn, Z. Wang, M. Hirschberger, N. P. Ong, and B. A. Bernevig, Chiral anomaly factory: Creating Weyl fermions with a magnetic field, Phys. Rev. B 95, 161306(R) (2017).

[16] S. Borisenko, D. Evtushinsky, Q. Gibson, A. Yaresko, K. Koepernik, T. Kim, M. Ali, J. van den Brink, M. Hoesch, A. Fedorov et al., Time-reversal symmetry breaking type-II Weyl state in $\mathrm{YbMnBi}_{2}$, Nat. Commun. 10, 3424 (2019).

[17] S. Chikazumi and C. D. Graham, Physics of Ferromagnetism, 2nd ed., Vol. 94 (Oxford University Press, Oxford, UK, 2009).

[18] J. W. Drijver, S. G. Sinnema, and F. Van der Woude, Magnetic properties of hexagonal and cubic $\mathrm{Fe}_{3} \mathrm{Ge}$, J. Phys. F 6, 2165 (1976). 
[19] F. Albertini, D. Negri, L. Pareti, E. B. Watts, Z. Arnold, J. Kamarad, G. Calestani, A. Deriu, and S. Besseghini, Magnetocrystalline anisotropy of $\mathrm{Fe}_{3} \mathrm{Ge}$ single crystal: Effect of pressure and Mn substitution for Fe, J. Appl. Phys. 96, 2110 (2004).

[20] Z. Lin, M. Lohmann, Z. A. Ali, C. Tang, J. Li, W. Xing, J. Zhong, S. Jia, W. Han, S. Coh, W. Beyermann, and J. Shi, Pressure-induced spin reorientation transition in layered ferromagnetic insulator $\mathrm{Cr}_{2} \mathrm{Ge}_{2} \mathrm{Te}_{6}$, Phys. Rev. Mat. 2, 051004 (2018).

[21] J. Lyubina, I. Opahle, K.-H. Müller, O. Gutfleisch, M. Richter, M. Wolf, and L. Schultz, Magnetocrystalline anisotropy in L10 $\mathrm{FePt}$ and exchange coupling in $\mathrm{FePt} / \mathrm{Fe}_{3} \mathrm{Pt}$ nanocomposites, J. Phys.: Condens. Matter 17, 4157 (2005).

[22] E. Liu, Y. Sun, N. Kumar, L. Muechler, A. Sun, L. Jiao, S.-Y. Yang, D. Liu, A. Liang, Q. Xu et al., Giant anomalous Hall effect in a ferromagnetic kagome-lattice semimetal, Nat. Phys. 14, 1125 (2018).

[23] Q. Wang, Y. Xu, R. Lou, Z. Liu, M. Li, Y. Huang, D. Shen, H. Weng, S. Wang, and H. Lei, Large intrinsic anomalous Hall effect in half-metallic ferromagnet $\mathrm{Co}_{3} \mathrm{Sn}_{2} \mathrm{~S}_{2}$ with magnetic Weyl fermions, Nat. Commun. 9, 3681 (2018).

[24] N. Morali, R. Batabyal, P. K. Nag, E. Liu, Q. Xu, Y. Sun, B. Yan, C. Felser, N. Avraham, and H. Beidenkopf, Fermiarc diversity on surface terminations of the magnetic Weyl semimetal $\mathrm{Co}_{3} \mathrm{Sn}_{2} \mathrm{~S}_{2}$, Science 365, 1286 (2019).

[25] D. F. Liu, A. J. Liang, E. K. Liu, Q. N. Xu, Y. W. Li, C. Chen, D. Pei, W. J. Shi, S. K. Mo, P. Dudin et al., Magnetic Weyl semimetal phase in a Kagomé crystal, Science 365, 1282 (2019).

[26] H. P. J. Wijn, 3d, 4d and 5d Elements, Alloys and Compounds (Springer-Verlag, Berlin, 1986).

[27] J. Trygg, B. Johansson, O. Eriksson, and J. M. Wills, Total Energy Calculation of the Magnetocrystalline Anisotropy Energy in the Ferromagnetic $3 d$ Metals, Phys. Rev. Lett. 75, 2871 (1995).

[28] The estimation is based on the MAE (experimental value for hcp-Co and our DFT value for $\mathrm{Co}_{3} \mathrm{Sn}_{2} \mathrm{~S}_{2}$ ) and on the leading term in the anisotropy $E=K_{0} \sin ^{2}(\theta)-M H \sin (\theta)$ where $M=$ $|\mathbf{m}|$ and $H$ is the external field [17]. For hcp-Co, $\mathbf{m}$ is about $1.6 \mu_{B}$ per atom (low $T$ ). For $\mathrm{Co}_{3} \mathrm{Sn}_{2} \mathrm{~S}_{2}$, the result agrees with magnetization measurements which indicate that at $10 \mathrm{~K}$ a field of 22.9 Tesla is required to make the in-plane magnetization reach the value presented by the out-of-plane component at zero field. We did not consider effects of the external field other than the rotation of $\mathbf{m}$.

[29] We used the all-electron full-potential local-orbital (FPLO) code [52] (18.00-52) in the fully relativistic mode using GGA (PBE-96) [53] with an enhanced basis set [54] and a linear tetrahedron method with $40 \times 40 \times 40$ for Co (and $12 \times 12 \times$ 12 for $\mathrm{Co}_{3} \mathrm{Sn}_{2} \mathrm{~S}_{2}$ ) intervals in the Brillouin zone.

[30] For the search of Weyl points, we used PYFPLO. For each $\theta$, we built a Hamiltonian by projecting the Bloch states onto atomicorbital-like Wannier functions of Co $3 d, 4 s$, and $4 p$ states for hep-Co and Co $3 d$ and $4 s$, Sn $5 p$ and $5 s$, and $\mathrm{S} 3 p$ for $\mathrm{Co}_{3} \mathrm{Sn}_{2} \mathrm{~S}_{2}$.

[31] See Supplemental Material at http://link.aps.org/supplemental/ 10.1103/PhysRevResearch.1.032044 for (i) hcp-Co Weyl nodes trajectories for different functionals, (ii) experimental estimation of the field needed to rotate $\mathbf{m}$ in $\mathrm{Co}_{3} \mathrm{Sn}_{2} \mathrm{~S}_{2}$, (iii) Berry curvature flux for different orientations of $\mathbf{m}$, (iv) $\mathrm{Co}_{3} \mathrm{Sn}_{2} \mathrm{~S}_{2}$ Weyl- node trajectories in the full Brillouin zone, and (v) derivation of Eq. (3) and further details about the numerical calculations.

[32] W. Schnelle, A. Leithe-Jasper, H. Rosner, F. M. Schappacher, R. Pöttgen, F. Pielnhofer, and R. Weihrich, Ferromagnetic ordering and half-metallic state of $\mathrm{Sn}_{2} \mathrm{Co}_{3} \mathrm{~S}_{2}$ with the shanditetype structure, Phys. Rev. B 88, 144404 (2013).

[33] P. Vaqueiro and G. G. Sobany, A powder neutron diffraction study of the metallic ferromagnet $\mathrm{Co}_{3} \mathrm{Sn}_{2} \mathrm{~S}_{2}$, Solid State Sci. 11, 513 (2009).

[34] Close to $\theta=\mathrm{v}$ there are actually two sets of Weyl points reaching the Fermi surface. In Fig. 3(v), for simplicity we chose to show the dispersion of only one of these sets. Also, we omit in Fig. 3 four Weyl nodes in the plane $k_{y}=0$ that are stable only in a small angle range close to $\pi / 2$.

[35] Y. D. Chong, Berry's phase and the anomalous velocity of Bloch wave packets, Phys. Rev. B 81, 052303 (2010).

[36] X. Wang, D. Vanderbilt, J. R. Yates, and I. Souza, Fermi-surface calculation of the anomalous Hall conductivity, Phys. Rev. B 76, 195109 (2007)

[37] D. Xiao, Y. Yao, Z. Fang, and Q. Niu, Berry-phase effect in anomalous thermoelectric transport, Phys. Rev. Lett. 97, 026603 (2006).

[38] C. Zhang, S. Tewari, V. M. Yakovenko, and S. Das Sarma, Anomalous Nernst effect from a chiral $d$-density-wave state in underdoped cuprate superconductors, Phys. Rev. B 78, 174508 (2008).

[39] R. Lundgren, P. Laurell, and G. A. Fiete, Thermoelectric properties of Weyl and Dirac semimetals, Phys. Rev. B 90, 165115 (2014).

[40] G. Sharma, P. Goswami, and S. Tewari, Nernst and magnetothermal conductivity in a lattice model of Weyl fermions, Phys. Rev. B 93, 035116 (2016).

[41] G. Y. Guo, Q. Niu, and N. Nagaosa, Anomalous Nernst and Hall effects in magnetized platinum and palladium, Phys. Rev. B 89, 214406 (2014).

[42] Y. Ferreiros, A. A. Zyuzin, and J. H. Bardarson, Anomalous Nernst and thermal Hall effects in tilted Weyl semimetals, Phys. Rev. B 96, 115202 (2017).

[43] T. M. McCormick, R. C. McKay, and N. Trivedi, Semiclassical theory of anomalous transport in type-II topological Weyl semimetals, Phys. Rev. B 96, 235116 (2017).

[44] S. Saha and S. Tewari, Anomalous Nernst effect in type-II Weyl semimetals, Eur. Phys. J. B 91, 4 (2018).

[45] E. V. Gorbar, V. A. Miransky, I. A. Shovkovy, and P. O. Sukhachov, Anomalous transport properties of Dirac and Weyl semimetals, Low Temp. Phys. 44, 487 (2018).

[46] A. A. Burkov, Weyl metals, Annu. Rev. Condens. Matter Phys. 9, 359 (2018)

[47] X. Li, L. Xu, L. Ding, J. Wang, M. Shen, X. Lu, Z. Zhu, and K. Behnia, Anomalous Nernst and Righi-Leduc Effects in $\mathrm{Mn}_{3} \mathrm{Sn}$ : Berry Curvature and Entropy Flow, Phys. Rev. Lett. 119, 056601 (2017).

[48] For numerical integration of Eq. (1), we used a mesh of density $400^{3} / V_{B Z}$ where $V_{B Z}$ is the Brillouin zone volume. The results of entropy-flow density were obtained with a density of $1500^{3} / V_{B Z}$ points and considering states in the energy window $[-20,20]$ $\mathrm{meV}$ [31].

[49] A. A. Burkov, Anomalous Hall Effect in Weyl Metals, Phys. Rev. Lett. 113, 187202 (2014).

[50] M. Ikhlas, T. Tomita, T. Koretsune, M.-T. Suzuki, D. Nishio- 
Hamane, R. Arita, Y. Otani, and S. Nakatsuji, Large anomalous Nernst effect at room temperature in a chiral antiferromagnet, Nat. Phys. 13, 1085 (2017).

[51] A. Sakai, Y. P. Mizuta, A. A. Nugroho, R. Sihombing, T. Koretsune, M.-T. Suzuki, N. Takemori, R. Ishii, D. NishioHamane, R. Arita et al., Giant anomalous Nernst effect and quantum-critical scaling in a ferromagnetic semimetal, Nat. Phys. 14, 1119 (2018).
[52] K. Koepernik and H. Eschrig, Full-potential nonorthogonal local-orbital minimum-basis band-structure scheme, Phys. Rev. B 59, 1743 (1999).

[53] J. P. Perdew, K. Burke, and M. Ernzerhof, Generalized Gradient Approximation Made Simple, Phys. Rev. Lett. 77, 3865 (1996).

[54] K. Lejaeghere, G. Bihlmayer, T. Björkman, P. Blaha, S. Blügel, V. Blum, D. Caliste, I. E. Castelli, S. J. Clark, A. Dal Corso et al., Reproducibility in density functional theory calculations of solids, Science 351, aad3000 (2016). 